\title{
A Study on Locations of Death and Factors Associated with Death among Cancer Patients in South Korea
}

\author{
Yong Joo Rhee',2*, Yoon-Hee Tae ${ }^{3}$, Yong Joo Lee ${ }^{4}$, Soomok Jang ${ }^{3}$, Joachim Cohen ${ }^{5}$, \\ Young-Soon $\mathrm{Choi}^{3}$
}

\author{
${ }^{1}$ Department of Health Sciences, Dongduk Women's University, Seoul, South Korea \\ ${ }^{2}$ Department of Psychiatry and Behavioral Sciences, Feinberg School of Medicine, Northwestern University, Chicago, Illinois, USA \\ ${ }^{3}$ National Health Insurance Service, Wonju, Kangwon-do, South Korea \\ ${ }^{4}$ Department of Palliative Medicine, Seoul St. Mary's Hospital, College of Medicine, The Catholic University of Korea, \\ Banpo-daero, Seocho-gu, Seoul, South Korea \\ ${ }^{5}$ Vrije Universiteit Brussel (VUB) \& Ghent University, End-of-Life Care Research Group, Brussels, Belgium \\ Email: ^yrh759@dongduk.ac.kr, *y-rhee@northwestern.edu
}

How to cite this paper: Rhee, Y.J., Tae, Y.-H., Lee, Y.J., Jang, S., Cohen, J. and Choi, Y.-S. (2019) A Study on Locations of Death and Factors Associated with Death among Cancer Patients in South Korea. Journal of Biosciences and Medicines, 7, 26-41.

https://doi.org/10.4236/jbm.2019.72003

Received: December 13, 2018

Accepted: January 20, 2019

Published: January 23, 2019

Copyright $\odot 2019$ by author(s) and Scientific Research Publishing Inc. This work is licensed under the Creative Commons Attribution International License (CC BY 4.0).

http://creativecommons.org/licenses/by/4.0/

\section{(c) (i) Open Access}

\begin{abstract}
Background: Location of death has been used to examine an indicator for good death. This study aims to examine location of death among patients with three major cancers (gastric, liver, and lung) and other factors associated with location of death in South Korea. Methods: We selected the medical and pharmacy claims data for health services and location of death among the 42,596 decedents with cancer (lung 16,632, liver 15,872, gastric 10,092) from 2009 to 2013. We used logistic regressions to identify factors associated with home death. Outcome measures are locations of death (hospital, outpatient clinics or emergency room and home). Results: Only $8.9 \%$ died at home whereas $46.5 \%$ died in hospital as inpatients. Patients with more than one comorbid cancer or receivers for any supportive care were significantly more likely to die in hospital. Female and younger than 55 years old liver cancer patients were associated with home death. Patients living in metropolitan area, or paying more insurance premium, or being public aid beneficiaries, were associated with home death. Conclusions: The supportive care service use prior to death was significantly associated with increasing odds to hospital death. Being older than 75 , or having multiple cancers was significant factors associated with hospital death, whereas living in metropolitan area, lower income or emergency visit were significant factors with home death. These findings are opposite to what is found, as the palliative care and hospice is predominantly hospital-centered. The findings emphasize a need to available end-of-life care in community for dying patients.
\end{abstract}




\section{Keywords}

Supportive Care, Location of Death, National Health Insurance Program (NHI), Cancer, End-of-Life Care

\section{Introduction}

A death at home has been considered as a significant outcome and a "good death" in a large body of research in end of life care [1] [2] [3] [4] [5] due to better quality of care [2] [6] and autonomy [3] [5] [6]. The main body of literature shows that people prefer to die at home [5] [7] [8] [9] irrespective of their health conditions and living arrangements prior to death [7] [8].

Several studies have suggested that palliative care provision may improve the chances of people to receive care in the final days outside hospital or at home and to die there [8] [10] [11] [12] [13]. Cancer patients are more likely to receive hospice and palliative care compared to other dying patients, both in hospital settings and at home, and their chances of achieving a chosen home death are better [14] [15] [16] [17]. Also, hospice and palliative care use were associated with home death for cancer patients in European countries [1] [11]. However, South Korea has a different situation. The majority of hospice and palliative care is being provided in hospital in South Korea, and this may be one of the explanations for the relatively high rate of hospital deaths (87.2\%) among cancer patients in South Korea [18]. If the hospital-focused development of palliative care in South Korea is indeed an explanation for the large proportion dying in hospital [19], this could also imply that those cancer patients get a supportive type of care at the end-of-life, which can include use of services such as hospice and palliative care and die so in the hospital rather than at home [20].

This study, therefore, aims to examine location of death use among patients with three major cancers (gastric, liver, and lung) and its association with home death in South Korea. These three cancers have been reported as the three major cancers for causes of deaths which took up over half among all cancers in South Korea [20].

This study has the following research questions:

1) What is the prevalence of home death among patients with three major cancers in South Korea?

2) What other factors are associated with home death among patients with three cancers in South Korea?

\section{Methods}

\subsection{Data and Study Cohort}

This study used medical and pharmacy claims data from the National Health Insurance (NHI) of decedents in 44 tertiary general hospitals from January $1^{\text {st }}$, 2009 to December 31, 2013 in South Korea. The dataset included all medical and 
pharmacy claims for health services uses and eligibility information of gender, age and insurance premium level. The National Health Insurance Service (NHIS), the single public payer, has full authority to access all deaths for all citizens through the electronic claims dataset system in South Korea.

Among the initial records for all 84,462 deaths, we developed the analytic dataset for this study limiting cases were diagnosed, and died of the major three cancers: gastric (C16), liver (C22), or lung (C34) cancers as a primary cause. The initial claims data were then used to identify decedents diagnosed with these three cancers as a primary diagnosis and attendant treatment during the last month prior to death. We only examined the last episode claim per decedent that was equal to the number of decedents. Thus, one last record for deaths diagnosed and died of these three major cancers (gastric (C16), liver (C22), lung (C34) cancers) as a primary cause were selected in this study. The NHIS provided the de-identified subset blinded medical and pharmacy claims data for this research project extracted from their own Hospice and Palliative Promotion project dataset. The Institutional Review Board at NHIS Ilsan Hospital already approved for the previous Hospice and Palliative Promotion project (IRB number \#3-1725-A-N-01) at NHIS before conducting this research project. Therefore, there is no need for any extra Institutional Review Board approval nor informed consents for our research project.

\subsection{Measures}

\subsubsection{Dependent Variable: Locations of Death}

Place of death comprised the categories of hospital, outpatient clinics or emergency room and home. There are two kinds of datasets for these decedents: the medical services claims and reports for death. Comparing these two datasets, we identified the locations of death (hospital, clinic or emergency room) if the last date for medical record (i.e. inpatient, outpatient clinic, or emergency room) was identical to that of the death report. Due to no field for the place of death itself in these datasets, we used the operational definition for "home death" if patient with cancer was not identified to die in the above three medical settings (hospital, outpatient clinic or emergency room). We assumed that cancer patients died at home if the last date for medical records were not matched with the dates of death report. Using current two datasets, we found the nursing home death would be extremely rare and was difficult to differentiate with hospital death. Most of nursing home death were coded in "hospital". Therefore, we did not differentiate the nursing home deaths with hospital death in this study.

\subsubsection{Independent Variables}

\section{Supportive Care Use}

We identified the uses of supportive care services if there were claim records reimbursed for palliative care unit, aseptic room patient care, lead-shield room patient care, isolation rooms and medications (narcotic pain pills, antiemetic agent for anti-cancer drug). These services are defined as non-aggressive treat- 
ment that does not aim for cure in this study so we defined as "supportive" care. When we found any reimbursed claims for chemotherapy, immunotherapy, basic tests for treatments (i.e. PET-CT, bone densitometry) in the last month of life, we defined these services for aggressive treatment for cancer. However, we excluded these cases for supportive care use, associated with treatment purpose procedure or test in this study.

Independent variables were age (0 - 40, 41 - 55, 56 - 64, 65 - 74, 75 - 84, 85 years+), sex (female, male), insurance type (the employee insured, the self-employed insured, public aid), and degree of urbanization of the municipality of residence (metropolitan city (population $\geq 1$ million, city $(1$ million $>$ population $\geq$ 30,000 ), and rural). In South Korea, employees pay their contributions based on income under the National Health Insurance Program, whereas the self-employed do on the basis of income and asset. The public aid beneficiaries are low income bracket and do not pay any contribution under the public aid program which is subsidized by the government tax. The degrees of their monthly contributions were evenly distributed and categorized into 20 groups (the lowest grade 1 to the highest grade 20) excluding the public aid beneficiaries. This rank was used as proxy information for beneficiaries' economic status such as income. The extents of health care services use were analyzed using the number of emergency visits in the last episode. We used the total number of cancers as comorbid condition as we could not differentiate clearly the primary and secondary cancers in medical claims. We did not consider other comorbid chronic disease as comorbid condition as these patients were severely dying of cancer in this study (i.e. hypertension, diabetes).

\subsection{Statistical Analysis}

We analyzed data for three groups separately for patients with gastric cancer, liver cancer and lung cancer.

For each patient with gastric cancer, liver cancer and lung cancer separately, a multivariable logistic regression model was constructed to determine independent predictors of home death. As patients with these three cancers have different paths in prognosis or pain levels at the last days of lives, we ran the model s individually. Due to big sample sizes for each group from the national datasets, statistical tests may be able to present meaningful results with significant power. Therefore, we developed the model s to examine interactions factors in the separate models.

The independent variables in each model were age, sex, urbanization, health care services use (ER visit) prior to death, severity of cancer (number of cancers), and level of monthly insurance contribution group.

To investigate associations between home death and factors which contributed to health services use effects, two sets of multivariable logistic regression model $s$ were performed. Using home death as the dependent variable, we developed two model s consisting of all independent variables for each insurance type (model 1), and then adding interactions; emergency visits and monthly 
contribution level, emergency visit and insurance type and female and monthly contribution group (model 2) in order to gain more insight on the respective role of these covariates. All model s were checked for multicollinearity (tolerance and variance inflation factor collinearity statistics). Model 2 was designed to re-examine the associations among higher medical utilizations, severity of cancers, economic levels, and home death after running model 1. All analyses were conducted using SAS 9.3.version (SAS Institute Inc., Cary, North Carolina).

\section{Results}

\subsection{Descriptive Statistics}

Table 1 shows that descriptive statistics of demographics or health services use among those who died of three major cancers (gastric, liver and lung cancer) in 44 tertiary hospitals. Among decedents with three major cancers total $42,596 \mathrm{pa}-$ tients (lung 16,632, liver 15,872, gastric 10,092) were identified. $71.6 \%$ of decedents were male. Their mean of age was 64.0 years and most lived in urban area (87.3\%). Over half of them were covered by any employer's insurance. The most frequent comorbid chronic diseases were diabetes among liver patients (14.4\%), lung patient (9.7\%) and gastric patients (9.3\%). The next frequent comorbid chronic disease was hypertension among three major cancer patients (lung, $6.8 \%$, liver, $6.0 \%$, gastric cancer, $5.7 \%$ ).

Only $1.7 \%$ of liver cancer patients received only supportive care services whereas $4.1 \%$ of patients with gastric cancer received only supportive care services prior to death. The most frequently received health services were pain management (8.2\%) and chemotherapy (6.5\%). Gastric cancer patients $(9.5 \%)$ used more pain management medication while $6.7 \%$ of patients with liver cancer used medication for pain management. More than $90 \%$ died in hospital: less than half (45.6\%) in the inpatient hospital and an almost equal proportion in the outpatient hospital or emergency room; 8.9\% died at home.

Over half of decedents had employer insurance among all patients while 8.0\% of decedents were covered by medical aids.

Among all 42,596 deaths, only $8.9 \%$ died outside of hospital or outpatients' clinics. Most of death occurred in inpatient setting (46.5\%).

\subsection{Factors Associated with Home Death among Patients with Three Cancers}

\subsubsection{Age and Gender}

Table 2 presents the logistic regressions results among patients who died of gastric, liver, and lung cancer. Table 3 includes the logistic regression results with the interaction variables.

Among gastric cancer patients, those aged between 65 and 74 were significantly to die at hospital, clinics or emergency room $(\mathrm{OR}=0.575, \mathrm{p}<0.01$, model 1) or patients those aged between 75 to 84 were less likely to die in any places in hospital. $(\mathrm{OR}=0.668, \mathrm{p}<0.05$, model 1$)$ in Table 2. Patients aged $41-55$ with 
Table 1. Descriptive statistics among patients with major three cancers prior to death.

\begin{tabular}{|c|c|c|c|c|c|c|c|c|c|}
\hline & & \multicolumn{2}{|c|}{$\begin{array}{l}\text { Gastric Cancer } \\
\text { (C16) }\end{array}$} & \multicolumn{2}{|c|}{$\begin{array}{l}\text { Liver Cancer } \\
\text { (C22) }\end{array}$} & \multicolumn{2}{|c|}{$\begin{array}{l}\text { Lung Cancer } \\
\text { (C34) }\end{array}$} & \multicolumn{2}{|c|}{ Total } \\
\hline & & $\mathrm{N}$ & $\%$ & $\mathrm{~N}$ & $\%$ & $\mathrm{~N}$ & $\%$ & $\mathrm{~N}$ & $\%$ \\
\hline & Total & 10,092 & 100 & 15,872 & 100 & 16,632 & 100 & 42,596 & 100 \\
\hline \multirow[t]{2}{*}{ Gender } & Female & 3240 & 32.1 & 3630 & 22.9 & 3846 & 23.1 & 10,716 & 25.2 \\
\hline & male & 6852 & 67.9 & 12,242 & 77.1 & 12,786 & 76.9 & 31,880 & 74.8 \\
\hline \multirow[t]{6}{*}{ Age group } & $0-40 \mathrm{yrs}$ & 709 & 7.0 & 533 & 3.4 & 286 & 1.7 & 1528 & 3.6 \\
\hline & $41-55 \mathrm{yrs}$ & 2294 & 22.7 & 4190 & 26.4 & 1903 & 11.4 & 8387 & 19.7 \\
\hline & $56-64$ yrs & 2407 & 23.9 & 4833 & 30.4 & 4089 & 24.6 & 11,329 & 26.6 \\
\hline & $65-74$ yrs & 2726 & 27.0 & 4229 & 26.6 & 6317 & 38.0 & 13,272 & 31.2 \\
\hline & $75-84$ yrs & 1666 & 16.5 & 1822 & 11.5 & 3521 & 21.2 & 7009 & 16.5 \\
\hline & 85 yrs \& older & 290 & 2.9 & 265 & 1.7 & 516 & 3.1 & 1071 & 2.5 \\
\hline Age (means) & & \multicolumn{2}{|c|}{62.6} & \multicolumn{2}{|c|}{61.8} & \multicolumn{2}{|c|}{67.2} & \multicolumn{2}{|c|}{63.9} \\
\hline Region & Metropolitan City & 5471 & 54.2 & 8716 & 54.9 & 8652 & 52.0 & 22,839 & 53.6 \\
\hline & City & 3400 & 33.7 & 5207 & 32.8 & 5586 & 33.6 & 14,193 & 33.3 \\
\hline & Rural & 1217 & 12.1 & 1940 & 12.2 & 2386 & 14.3 & 5543 & 13.0 \\
\hline & unclear & 4 & 0.0 & 9 & 0.1 & 8 & 0.0 & 21 & 0.0 \\
\hline \multirow[t]{21}{*}{$\begin{array}{l}\text { The grade of monthly } \\
\text { contribution }\end{array}$} & unclear & 380 & 3.8 & 586 & 3.7 & 527 & 3.2 & 1493 & 3.6 \\
\hline & 1 grade $(0 \%-5 \%$ rank $)$ & 279 & 2.8 & 411 & 2.6 & 508 & 3.1 & 1198 & 2.8 \\
\hline & 2 grade $(6 \%-10 \%$ rank $)$ & 317 & 3.1 & 484 & 3.0 & 451 & 2.7 & 1252 & 2.9 \\
\hline & 3 grade $(11 \%-15 \%$ rank $)$ & 266 & 2.6 & 492 & 3.1 & 485 & 2.9 & 1243 & 2.9 \\
\hline & 4 grade $(16 \%-20 \%$ rank $)$ & 253 & 2.5 & 472 & 3.0 & 427 & 2.6 & 1152 & 2.7 \\
\hline & 5 grade $(21 \%-25 \%$ rank $)$ & 262 & 2.6 & 430 & 2.7 & 420 & 2.5 & 1112 & 2.6 \\
\hline & 6 grade $(26 \%-30 \%$ rank $)$ & 274 & 2.7 & 470 & 3.0 & 460 & 2.8 & 1204 & 2.8 \\
\hline & 7 grade $(31 \%-35 \%$ rank $)$ & 325 & 3.2 & 522 & 3.3 & 543 & 3.3 & 1309 & 3.3 \\
\hline & 8 grade $(36 \%-40 \%$ rank $)$ & 383 & 3.8 & 525 & 3.3 & 575 & 3.5 & 1483 & 3.5 \\
\hline & 9 grade $(41 \%-45 \%$ rank $)$ & 347 & 3.4 & 627 & 4.0 & 562 & 3.4 & 1536 & 3.6 \\
\hline & 10 grade $(46 \%-50 \%$ rank $)$ & 410 & 4.1 & 607 & 3.8 & 629 & 3.8 & 1646 & 3.9 \\
\hline & 11 grade $(51 \%-55 \%$ rank $)$ & 420 & 4.2 & 649 & 4.1 & 647 & 3.9 & 1716 & 4.0 \\
\hline & 12 grade $(56 \%-60 \%$ rank $)$ & 409 & 4.1 & 734 & 4.6 & 702 & 4.2 & 1845 & 4.3 \\
\hline & 13 grade $(61 \%-65 \%$ rank $)$ & 440 & 4.4 & 712 & 4.5 & 656 & 3.9 & 1808 & 4.2 \\
\hline & 14 grade $(66 \%-70 \%$ rank $)$ & 442 & 4.4 & 681 & 4.3 & 737 & 4.4 & 1860 & 4.4 \\
\hline & 15 grade $(71 \%-75 \%$ rank $)$ & 525 & 5.2 & 788 & 5.0 & 784 & 4.7 & 2097 & 5.0 \\
\hline & 16 grade $(76 \%-80 \%$ rank $)$ & 475 & 4.7 & 823 & 5.2 & 944 & 5.7 & 2242 & 5.3 \\
\hline & 17 grade $(81 \%-85 \%$ rank $)$ & 696 & 6.9 & 957 & 6.0 & 1078 & 6.5 & 2731 & 6.4 \\
\hline & 18 grade $(86 \%-90 \%$ rank $)$ & 686 & 6.8 & 991 & 6.2 & 1179 & 7.1 & 2856 & 6.7 \\
\hline & 19 grade $(91 \%-95 \%$ rank $)$ & 606 & 6.0 & 895 & 5.6 & 1010 & 6.1 & 2511 & 5.9 \\
\hline & 20 grade $(96 \%-100 \%$ rank) & 1080 & 10.7 & 1663 & 10.5 & 2081 & 12.5 & 4824 & 11.3 \\
\hline
\end{tabular}


Continued

\begin{tabular}{|c|c|c|c|c|c|c|c|c|c|}
\hline Insurance type & Employer ${ }^{\wedge}$ & 5823 & 57.7 & 8860 & 55.8 & 10,013 & 60.2 & 24,696 & 60.0 \\
\hline & Self-employed & 3452 & 34.2 & 5659 & 35.7 & 5392 & 32.4 & 14,503 & 34.0 \\
\hline & Public Aid & 817 & 8.1 & 1353 & 8.5 & 1227 & 7.4 & 3397 & 8.0 \\
\hline Intensity of Medical Care & Non-aggressive care services ${ }^{\#}$ & 412 & 4.1 & 270 & 1.7 & 431 & 2.6 & 1113 & 2.6 \\
\hline \multirow[t]{3}{*}{ Number of ER visits } & & 5243 & 52.0 & 6890 & 43.4 & 7783 & 46.8 & 19,916 & 46.7 \\
\hline & 1 & 4425 & 43.8 & 8632 & 54.4 & 8349 & 50.2 & 21,406 & 50.3 \\
\hline & 2 & 1 & 0.0 & 2 & 0.0 & 3 & 0.0 & 6 & 0 \\
\hline \multirow[t]{5}{*}{ Number of Cancers } & 1 & 3381 & 33.5 & 10,992 & 69.3 & 7254 & 43.6 & 21,627 & 50.1 \\
\hline & 2 & 3493 & 34.6 & 3245 & 20.4 & 4,815 & 29.0 & 11,553 & 27.1 \\
\hline & 3 & 2015 & 20.0 & 1149 & 7.2 & 2,847 & 17.1 & 6011 & 14.1 \\
\hline & 4 & 869 & 8.6 & 366 & 2.3 & 1,189 & 7.1 & 2424 & 5.7 \\
\hline & 5 & 334 & 3.3 & 120 & 0.8 & 527 & 3.2 & 981 & 2.3 \\
\hline \multicolumn{10}{|l|}{ Comorbid Other Chronic } \\
\hline \multirow[t]{7}{*}{ Disease } & Hypertension & 579 & 5.7 & 946 & 6.0 & 1127 & 6.8 & 2652 & 6.2 \\
\hline & Diabetes & 936 & 9.3 & 2283 & 14.4 & 1609 & 9.7 & 4828 & 11.3 \\
\hline & Depression & 142 & 1.4 & 70 & 0.4 & 177 & 1.1 & 389 & 0.9 \\
\hline & Coronary Artery Disease (CAD) & 286 & 2.8 & 293 & 1.8 & 662 & 4.0 & 1241 & 2.9 \\
\hline & $\begin{array}{c}\text { Chronic Obstructive } \\
\text { Pulmonary Disease (COPD) }\end{array}$ & 254 & 2.5 & 230 & 1.5 & 2020 & 12.1 & 2504 & 5.8 \\
\hline & Renal failure & 114 & 1.1 & 221 & 1.4 & 173 & 1.0 & 508 & 1.2 \\
\hline & Others & 7666 & 75.6 & 11,778 & 74.2 & 9623 & 57.8 & 28,089 & 65.9 \\
\hline \multirow[t]{11}{*}{ Health Services } & $\mathrm{CT}$ & 212 & 2.1 & 297 & 1.9 & 359 & 2.2 & 868 & 2.0 \\
\hline & MRI & 28 & 0.3 & 42 & 0.3 & 95 & 0.6 & 165 & 0.4 \\
\hline & PET & 14 & 0.1 & 24 & 0.2 & 37 & 0.2 & 75 & 0.2 \\
\hline & Chemotherapy & 616 & 6.1 & 1121 & 7.1 & 1098 & 6.6 & 2835 & 6.7 \\
\hline & $\begin{array}{c}\text { Pain management } \\
\text { Pharmacotherapy (Cancer) }\end{array}$ & 956 & 9.5 & 1071 & 6.7 & 1478 & 8.9 & 3505 & 8.4 \\
\hline & $\begin{array}{l}\text { Cardiopulmonary } \\
\text { Resuscitation (CPR) }\end{array}$ & 11 & 0.1 & 16 & 0.1 & 22 & 0.1 & 49 & 0.1 \\
\hline & Psychotherapy & 5 & 0.0 & 4 & 0.0 & 10 & 0.6 & 19 & 0.0 \\
\hline & Intubation Use & 17 & 0.2 & 26 & 0.2 & 38 & 0.3 & 81 & 0.2 \\
\hline & Ventilator Use & 31 & 0.3 & 43 & 0.3 & 70 & 0.4 & 144 & 0.3 \\
\hline & Emergency Room Visit & 46 & 0.5 & 88 & 0.6 & 86 & 0.5 & 220 & 0.5 \\
\hline & Intensive Care Unit Use & 44 & 0.4 & 73 & 0.5 & 97 & 0.58 & 214 & 0.5 \\
\hline \multirow[t]{3}{*}{ Place of Death } & Inpatient Hospital & 5082 & 50.36 & 6488 & 40.9 & 7370 & 44.3 & 20,346 & 45.2 \\
\hline & Outpatient or Emergency Room & 4069 & 40.32 & 7992 & 50.5 & 7764 & 46.7 & 23,748 & 45.8 \\
\hline & Home & 941 & 9.32 & 1392 & 8.8 & 1498 & 9.0 & 4282 & 9.0 \\
\hline
\end{tabular}

*All information of demographics was described at the end of life time period. ^Employer insurance includes governments, public and private schools, and any private employers. "We used own operational definition of hospice and palliative care called "non-aggressive treatment" in this study for cases if there was no any aggressive treatments for cancer i.e. chemotherapy CT, MRI, PET, gene test or sonography. 
Table 2. Factors associated with home death among patients with three major cancers (Model 1).

\begin{tabular}{|c|c|c|c|c|c|c|c|}
\hline & & \multicolumn{2}{|c|}{ Gastric Cancer (C16) } & \multicolumn{2}{|c|}{ Liver Cancer (C22) } & \multicolumn{2}{|c|}{ Lung Cancer (C34) } \\
\hline & & Adjusted OR & $95 \%$ CI & Adjusted OR & $95 \%$ CI & Adjusted OR & $95 \%$ CI \\
\hline Gender & Female (vs. male) & 1.122 & $(0.958-1.315)$ & 1.124 & $(0.975-1.297)$ & $1.199^{* *}$ & $(1.043-1.378)$ \\
\hline \multirow[t]{6}{*}{ Age group } & $0-40$ & $\begin{array}{l}\text { Reference } \\
\text { category }\end{array}$ & & $\begin{array}{l}\text { Reference } \\
\text { category }\end{array}$ & & $\begin{array}{l}\text { Reference } \\
\text { category }\end{array}$ & \\
\hline & $41-55$ yrs & 0.896 & $(0.633-1.268)$ & $1.482^{*}$ & $(1.090-2.015)$ & $1.856^{* *}$ & $(1.226-2.808)$ \\
\hline & $56-64$ yrs & 0.714 & $(0.506-1.007)$ & 1.279 & $(0.943-1.735)$ & $1.605^{*}$ & $(1.085-2.372)$ \\
\hline & $65-74$ yrs & $0.575^{\star *}$ & $(0.410-0.806)$ & 1.135 & $(0.834-1.544)$ & 1.326 & $(0.903-1.947)$ \\
\hline & $75-84$ yrs & $0.668^{*}$ & $(0.467-0.956)$ & 0.983 & $(0.707-1.368)$ & 1.130 & $(0.764-1.671)$ \\
\hline & 85 yrs \& older & 0.769 & $(0.447-1.322)$ & 0.762 & $(0.470-1.234)$ & 0.931 & $(0.581-1.493)$ \\
\hline \multirow[t]{3}{*}{ Region } & Rural & $\begin{array}{l}\text { Reference } \\
\text { category }\end{array}$ & & $\begin{array}{l}\text { Reference } \\
\text { category }\end{array}$ & & $\begin{array}{l}\text { Reference } \\
\text { category }\end{array}$ & \\
\hline & Metropolitan City & $1.512^{* *}$ & $(1.231-1.857)$ & $1.270^{* *}$ & $(1.070-1.507)$ & $1.308^{* *}$ & $(1.121-1.527)$ \\
\hline & City & 1.181 & $(0.954-1.461)$ & 1.119 & $(0.934-1.341)$ & $1.236^{* *}$ & $(1.05-1.455)$ \\
\hline $\begin{array}{l}\text { The grade of } \\
\text { monthly contribution }\end{array}$ & Grade 1 - grade 20 & 1.010 & $(0.997-1.022)$ & $1.016^{* *}$ & $(1.006-1.027)$ & 1.011 & $(1.001-1.021)$ \\
\hline $\begin{array}{l}\text { Intensity of } \\
\text { Treatment }\end{array}$ & Supportive Care Use & $0.637^{\star *}$ & $(0.472-0.860)$ & $0.542^{* *}$ & $(0.383-0.768)$ & 0.939 & $(0.668-1.32)$ \\
\hline Insurance type & $\begin{array}{c}\text { Employer (vs. } \\
\text { community-based } \\
\text { insurance) }\end{array}$ & $1.180^{*}$ & $(1.023-1.361)$ & $1.230^{* *}$ & $(1.096-1.380)$ & $1.461^{* *}$ & $(1.306-1.635)$ \\
\hline Public aid & Medical Aid Beneficiary & $1.150^{* *}$ & $(1.072-1.235)$ & $1.146^{* *}$ & $(1.058-1.242)$ & $1.088^{* *}$ & $(1.03-1.15)$ \\
\hline Health Care & Number of ER visits & $1.197^{* *}$ & $(1.026-1.397)$ & 1.115 & $(0.983-1.266)$ & 1.120 & $(0.99-1.267)$ \\
\hline Comorbidity & Number of Cancers & $1.731^{* *}$ & $(0.555-0.964)$ & $0.690^{* *}$ & $(0.555-0.857)$ & $0.653^{* *}$ & $(0.525-0.812)$ \\
\hline \multirow[t]{3}{*}{ Interactions } & $\begin{array}{l}\text { ER visit }{ }^{*} \text { monthly } \\
\text { contribution }\end{array}$ & - & & - & & - & \\
\hline & ER visit ${ }^{*}$ Insurance type & - & & - & & & \\
\hline & $\begin{array}{l}\text { Female }{ }^{*} \text { monthly } \\
\text { contribution }\end{array}$ & - & & - & & - & \\
\hline
\end{tabular}

${ }^{\star} \mathrm{P}<0.05,{ }^{*} \mathrm{P}<0.001$, Model 1 : Logistic regression result without interactions.

Table 3. Factors associated with home death among patients with three major cancers (Model 2).

\begin{tabular}{|c|c|c|c|c|c|c|c|}
\hline & & \multicolumn{2}{|c|}{ Gastric Cancer (C16) } & \multicolumn{2}{|c|}{ Liver Cancer (C22) } & \multicolumn{2}{|c|}{ Lung Cancer (C34) } \\
\hline & & Adjusted OR & $95 \% \mathrm{CI}$ & Adjusted OR & $95 \% \mathrm{CI}$ & Adjusted OR & $95 \% \mathrm{CI}$ \\
\hline Gender & Female (vs. male) & $1.440^{*}$ & $(1.076-1.927)$ & 1.234 & $(0.961-1.584)$ & 1.186 & $(0.931-1.512)$ \\
\hline \multirow[t]{6}{*}{ Age group } & $0-40$ & $\begin{array}{c}\text { Reference } \\
\text { category }\end{array}$ & & $\begin{array}{l}\text { Reference } \\
\text { category }\end{array}$ & & $\begin{array}{c}\text { Reference } \\
\text { category }\end{array}$ & \\
\hline & $41-55 \mathrm{yrs}$ & 0.904 & $(0.638-1.28)$ & $1.483^{*}$ & $(1.091-2.016)$ & $1.857^{* * *}$ & $(1.227-2.810)$ \\
\hline & $56-64$ yrs & 0.719 & $(0.51-1.015)$ & 1.276 & $(0.941-1.731)$ & $1.606^{* *}$ & $(1.086-2.374)$ \\
\hline & $65-74$ yrs & $0.574^{* *}$ & $(0.409-0.805)$ & 1.129 & $(0.83-1.537)$ & 1.327 & $(0.903-1.949)$ \\
\hline & $75-84$ yrs & $0.667^{*}$ & $(0.466-0.955)$ & 0.982 & $(0.705-1.366)$ & 1.130 & $(0.764-1.672)$ \\
\hline & 85 yrs \& older & 0.761 & $(0.442-1.308)$ & 0.757 & $(0.467-1.227)$ & 0.932 & $(0.582-1.495)$ \\
\hline
\end{tabular}




\section{Continued}

\begin{tabular}{|c|c|c|c|c|c|c|c|}
\hline Region & Rural & $\begin{array}{c}\text { Reference } \\
\text { category }\end{array}$ & & $\begin{array}{l}\text { Reference } \\
\text { category }\end{array}$ & & $\begin{array}{c}\text { Reference } \\
\text { category }\end{array}$ & \\
\hline & Metropolitan City & $1.515^{\star *}$ & $(1.233-1.861)$ & $1.274^{* *}$ & $(1.073-1.512)$ & $1.309^{\star * *}$ & $(1.121-1.528)$ \\
\hline & City & 1.181 & $(0.954-1.461)$ & 1.122 & $(0.936-1.344)$ & $1.237^{* * *}$ & $(1.051-1.456)$ \\
\hline $\begin{array}{l}\text { The grade of monthly } \\
\text { contribution }\end{array}$ & Grade 1 - grade 20 & $1.024^{* *}$ & $(1.007-1.041)$ & 1.009 & $(0.994-1.023)$ & 1.012 & $(0.999-1.025)$ \\
\hline $\begin{array}{c}\text { Intensity } \\
\text { of Treatment }\end{array}$ & Supportive Care Use & $0.634^{* *}$ & $(0.47-0.856)$ & $0.543^{* *}$ & $(0.384-0.769)$ & 0.936 & $(0.666-1.316)$ \\
\hline Insurance type & $\begin{array}{l}\text { Employer (vs. } \\
\text { community-based } \\
\text { insurance) }\end{array}$ & $1.428^{*}$ & $(1.079-1.889)$ & 1.033 & $(0.832-1.282)$ & $1.420^{* *}$ & $(1.143-1.765)$ \\
\hline Public aid & Medical Aid Beneficiary & $1.151^{* *}$ & $(1.073-1.236)$ & $1.144^{* *}$ & $(1.056-1.24)$ & $1.088^{* *}$ & $(1.030-1.150)$ \\
\hline Health Care & Number of ER visits & 1.212 & $(0.992-1.481)$ & 1.033 & $(0.954-1.357)$ & 1.065 & $(0.905-1.253)$ \\
\hline Comorbidity & Number of Cancers & $0.741^{*}$ & $(0.561-0.977)$ & $0.692^{* *}$ & $(0.556-0.86)$ & $0.653^{* *}$ & $(0.525-0.813)$ \\
\hline \multirow[t]{3}{*}{ Interactions } & $\begin{array}{l}\text { ER visit }{ }^{*} \text { monthly } \\
\text { contribution }\end{array}$ & 0.983 & $(0.962-1.004)$ & $1.019^{*}$ & $(1.002-1.037)$ & 0.997 & $(0.980-1.014)$ \\
\hline & ER visit ${ }^{*}$ Insurance type & 0.982 & $(0.730-1.321)$ & 0.966 & $(0.761-1.225)$ & 1.119 & $(0.883-1.418)$ \\
\hline & $\begin{array}{l}\text { Female }{ }^{*} \text { monthly } \\
\text { contribution }\end{array}$ & $0.977^{*}$ & $(0.955-0.999)$ & 0.992 & $(0.972-1.011)$ & 1.001 & $(0.982-1.020)$ \\
\hline
\end{tabular}

${ }^{*} \mathrm{P}<0.05,{ }^{* *} \mathrm{P}<0.001$, Model 2: Logistic regressions results with interactions.

liver cancer $(\mathrm{OR}=1.482, \mathrm{p}<0.01$, model 1$)$ or lung cancer $(\mathrm{OR}=1.856, \mathrm{p}<$ 0.01 , model 1 ) were significantly more likely to die at home.

Female gastric cancer patients $(\mathrm{OR}=1.44, \mathrm{p}<0.05$, model 2, Table 3$)$, and female lung cancer patients ( $\mathrm{OR}=1.199, \mathrm{p}<0.05$, model 1 , Table 2 ) were significantly more likely to die at home comparing to male patients with gastric or lung cancer. Female gastric cancer patients paying for greater monthly premium contribution were significantly less likely to die at home $(\mathrm{OR}=0.977, \mathrm{p}<0.05$, model 2, Table 3).

\subsubsection{Urbanization}

All three cancer patients; gastric cancer patients $(\mathrm{OR}=1.512, \mathrm{p}<0.01$, model 1 , Table 2), liver cancer ( $\mathrm{OR}=1.27, \mathrm{p}<0.05$, model 1 , Table 2$)$ and lung cancer patients $(\mathrm{OR}=1.308 . \mathrm{p}<0.01$, model 1 , Table 2$)$ who lived in metropolitan area were significantly more likely to die at home than those who lived in rural area.

\subsubsection{Insurance Types and Monthly Premium Contribution Level}

Among those had employer insurance, gastric cancer patients $(\mathrm{OR}=1.18, \mathrm{p}<$ 0.01 , model 1, Table 2) and liver cancer patients $(\mathrm{OR}=1.23, \mathrm{p}<0.01$, model 1 , Table 2) and lung cancer patients $(\mathrm{OR}=1.461, \mathrm{p}<0.01$, Table 2$)$ were significantly more likely to die at home.

Patients who paid more monthly premium were significantly more likely to die at home among gastric cancer $(\mathrm{OR}=1.024, \mathrm{p}<0.01$, model 2 , Table 3$)$, liver 
patients $(\mathrm{OR}=1.016, \mathrm{p}<0.01$, model 1 , Table 2$)$, and lung patients $(\mathrm{OR}=1.011$, $\mathrm{p}<0.05$, model 1, Table 2).

Medical aid beneficiaries with gastric cancer $(\mathrm{OR}=1.150, \mathrm{p}<0.01$, model 1 , Table 2 ) or liver cancer $(\mathrm{OR}=1.146, \mathrm{p}<0.01$, model 1 , Table 2$)$ or lung cancer $(\mathrm{OR}=1.088, \mathrm{p}<0.01$, model 1 , Table 2$)$ were significantly more likely to die at home.

\subsubsection{Supportive Care Use}

Using only supportive care was significantly associated with death in any ward in hospital such as hospital room, emergency room or clinics among gastric cancer patients $(\mathrm{OR}=0.637, \mathrm{p}<0.01$, model 1 and liver cancer patients $(\mathrm{OR}=$ $0.542, \mathrm{p}<0.01$, model 1)) in Table 2.

\subsubsection{Emergency Room Visit and Total Number of Cancers}

If gastric cancer patients $(\mathrm{OR}=1.197, \mathrm{p}<0.01$, model 1$)$ or liver cancer patients $(\mathrm{OR}=1.115, \mathrm{p}<0.05$, model 1$)$ or lung cancer patients $(\mathrm{OR}=1.120, \mathrm{p}<0.05$, model 1) had a higher number of emergency room visits in the last month of life, they had significantly higher probabilities to die at home. The interaction factor between emergency room visit and monthly premium contribution level was significantly associated with home death among liver cancer patients $(\mathrm{OR}=$ 1.019, $\mathrm{p}<0.05$, model 2, Table 3).

All patients with greater numbers of comorbid cancer were significantly associated with hospital death. In particular, gastric cancer patients with other comorbid cancer had lower odds to die at home (OR $=0.743 \mathrm{p}<0.001$, model 2, Table 3).

\section{Discussion}

Most of patients with any cancer in South Korea are more likely to die in hospital (87.2\%) which is surprisingly greater than other countries [18]. Compared to other countries, there are particular factors associated with hospital death related to the end-of-life care. Among them, lack of home hospice care service is most attributing factor of hospital death in terminal cancer patients. To improve quality of death, Korean medical system needs to develop home hospice care service.

This study examined the location of death among the major three cancer patients (gastric, liver and lung) using medical claims dataset and examined the factors associated with home death. This study is the first empirical study using the national level of claims data for the decedents with major three cancers in South Korea and examined the death places. Those who received only supportive care in the last month of life were significantly more likely to die in any places in hospital. If patients with more than one cancer had greater probability to die in hospital, living in a metropolitan area, being female among gastric and lung cancer patients, or being younger was associated with home death.

Considering the quality of life among the dying patients and preferences, it is clear that most people have preferred their home as the place of death over a bed 
in hospital [21] [22] [23] and dying home is ideal for the dying individuals and their families [24] [25]. However, the prevalence of home death is still lower [25] [26]. South Korean situation is also not exceptional, either. Majority of descendants do die in hospital although the Koreans prefer to die at home [27] [28].

Location of death and its factors have been studied in diverse the advanced end-of-life health system. In Japan, availabilities for hospice or palliative care at home were significantly associated with home death better psychological status for patients and families [20] [29] [30]. Also, Japanese patients had greater change to die at home without any preferred place for death [31]. Diverse studies revealed the factors associated with home death: caregivers' preferred place, caregivers' support, numbers of hospital admissions, palliative care visits [33] [34], being married, higher educations, and urbanization [11]. However, dying patient with greater need for medical attention (i.e. pain management) had high probability of dying in hospital. So, if pain is controllable at home, there would be greater chances to let the patients die at home [30] [31].

Urbanization factor implies the access for medical services. Living in metropolitan area increases access to hospital often so it is easy to discharge and to be readmitted to hospitals whereas residents in rural or small cities.

Therefore, supportive hospital care services increase the chance of hospital death rather than decreasing it, and living in metropolitan area increased the chance of home death. Being female, or living in metropolitan area was associated with hospital death in the previous study [32] [33].

We found that both decedents with higher income and low income who got supported by public aid were more likely to die at home interestingly. This finding rooted the two different reasons why these two groups led to home death; one with greater resource wanted to die at home for better death and the other with lower access to medical services or support might not be able to receive sufficient services at medical setting.

Hospice or palliative care is indeed strongly hospital-focused in South Korea. Palliative care at home is also available but it is a service provided by the hospital to patients that cannot be hospitalized for some reason and when a registered nurse is available [35] [36]. A majority of hospice and palliative care services in South Korea still concentrate on predominantly cancer patients in tertiary hospitals supported by the Cancer Control Policy [37]. The pilot project for home-based hospice and palliative care services have begun since 2016 March, which is an extension form of hospice and palliative care services provided by only certified hospitals [38].

The end-of-life care, hospice or palliative care services are being under-utilized in Korea still. In 2012, among those who died from cancer only, 11.9\% received palliative care from various institutions. In 2013, about 10\% (8084) cancer patients obtained services from palliative care institutions. These findings indicate that the current support system is still lacking, especially when compared other countries with hospice and palliative care under system. 


\section{Limitations}

This study has limitations. First, there was no exact information where the patients died except hospital, emergency room, or clinics. So home deaths may be overestimated in this study. There is no method to link with even the death certificate so we could not identify with the actual cause of death for our study sample. Due to the privacy act for patients, it is impossible to incorporate between the medical records and the death certificate. Thus, it might generate major inaccuracy for the place of death and it was impossible to validate the issues for this study.

Second, hospice or palliative care use was not clearly identified as an independent service code in the data system back then. The end-of-life care system is being in progress to add more services and payment system still during the study period. Even it is very controversial to use the terms hospice and palliative care at the same time for different stakeholders.

Despite these limitations, this study is a rare study to examine the locations of death as outcomes defined by operative definition using the large secondary claims data and its factors among patients with major cancers in South Korea. This effort reveals a few valuable findings to understand the patterns of health care services and significant factors associated with supportive care use among the dying patients.

\section{Conclusion}

Patients with three major cancers in South Korea were more likely to die at home if they lived metropolitan area, or were paying higher insurance premium, or were covered by employer insurance. The cancer patients who had supportive care services prior to death were significantly more likely to die in hospital in Korea. Patients with multi-cancers or being older had increased odds of dying in hospital. The end-of-life care system in Korea developed in hospital settings without the extensive or various formats of other choices for the dying patients often set the boundaries against the preferences of patients or family members still.

\section{Acknowledgements}

This study was a subset of Hospice Use and Application Research Study. The study was supported by the National Health Insurance Service and was coordinated by Dr. Choi and Ms. Tae in the Health Policy Research Team, the National Health Insurance System, South Korea.

\section{Ethics Approval and Consent to Participate}

Not applicable.

This research project was a subset of de-identified blinded medical and pharmacy claims data from the National Health Insurance Service, so there is no need for an Institutional Review Board approval nor informed consents to participate. 


\section{Consent to Publish}

Not applicable.

\section{Availability of Data and Materials}

Not applicable

\section{Funding}

This research is supported by a research fund of Korean Palliative Medicine Research Network (Grant Numbers KPMRNRF01601).

\section{Authors' Contributions}

YR: study concept, statistical analysis, interpretation of the data, drafting and revising the manuscript for content.

YT: study concept, statistical analysis, interpretation of the data, revising the manuscript for content, acquisition of data, study supervision, final approval of the version.

YC: study concept, interpretation of the data, revising of the manuscript for content, study supervision, final approval of the version of the manuscript to be submitted.

JC: Interpretation of the data, revising of the manuscript for content.

SJ: Interpretation of the data, revising of the manuscript for content.

YL: Interpretation of the data, revising of the manuscript for content.

YT and YC had full access to all of the data in the study and took responsibility for the integrity of the data and the accuracy of the data analysis. All authors read and approved the final manuscript.

\section{Conflicts of Interest}

The authors declared no potential conflicts of interest with respect to the research, authorship, and/or publication of this article.

\section{References}

[1] Ahlner-Elmqvist, M., et al. (2004) Place of Death: Hospital-Based Advanced Home Care versus Conventional Care: A Prospective Study in Palliative Cancer Care. Palliative Medicine, 18, 585-593. https://doi.org/10.1191/0269216304pm924oa

[2] Albrecht, J., Gruber-Baldini, A.L., Fromme, E.K., McGregor, J.C., Lee, D.S. and Furuno, J.P. (2013) Quality of Hospice Care for Individuals with Dementia. Journal of the American Geriatrics Society, 61, 1060-1065. https://doi.org/10.1111/jgs.12316

[3] Hoare, S., et al. (2015) Do Patients Want to Die at Home? A Systematic Review of the UK Literature, Focused on Missing Preferences for Place of Death. PLoS ONE, 10, 1-17. https://doi.org/10.1371/journal.pone.0142723

[4] Keam, B., et al. (2013) The Attitudes of Korean Cancer Patients, Family Caregivers, Oncologists and Members of the General Public toward Advance Directives. Supportive Care in Cancer, 21, 1437-1444. https://doi.org/10.1007/s00520-012-1689-z 
[5] Thomas, C., Morris, S.M. and Clark, D. (2004) Place of Death: Preferences among Cancer Patients and Their Carers. Social Science \& Medicine, 58, 2431-2444. https://doi.org/10.1016/j.socscimed.2003.09.005

[6] Evans, W.G., et al. (2006) Is There no Place like Home? Caregivers Recall Reasons for and Experience upon Transfer from Home Hospice to Inpatient Facilities. Journal of Palliative Medicine, 9, 100-110. https://doi.org/10.1089/jpm.2006.9.100

[7] Gomes, B., et al. (2013) Heterogeneity and Changes in Preferences for Dying at Home: A Systematic Review. BMC Palliative Care, 12, 12-17. https://doi.org/10.1186/1472-684X-12-7

[8] Guerriere, D., et al. (2015) Predictors of Place of Death for Those in Receipt of Home-Based Palliative Care Services in Ontario, Canada. Journal of Palliative Care, 31, 76-88. https://doi.org/10.1177/082585971503100203

[9] Schou-Andersen, M., Ullersted, M.P., Jensen, A.B. and Neergaard, M.A. (2016) Factors Associated with Preference for Dying at Home among Terminally Ill Patients with Cancer. Scandinavian Journal of Caring Sciences, 30, 466-476. https://doi.org/10.1111/scs.12265

[10] Houttekier, D., et al. (2010) Involvement of Palliative Care Services Strongly Predicts Place of Death in Belgium. Journal of Palliative Medicine, 13, 1461-1468. https://doi.org/10.1089/jpm.2010.0279

[11] Cohen, J., Houttekier, D., Onwuteaka-Philipsen, B., Miccinesi, G., et al. (2010) Which Patients with Cancer Die at Home? A Study of Six European Countries Using Death Certificate Data. Journal of Clinical Oncology, 28, 2267-2273. https://doi.org/10.1200/JCO.2009.23.2850

[12] Cohen, J., Houttekier, D., Chambaere, K., Bilsen, J. and Deliens, L. (2011) The Use of Palliative Care Services Associated with Better Dying Circumstances. Results from an Epidemiological Population-Based Study in the Brussels Metropolitan Region. Journal of Pain and Symptom Management, 42, 839-851.

https://doi.org/10.1016/j.jpainsymman.2011.02.017

[13] Wallace, S.K., Waller, D.K., Tilley, B.C., Piller, L.B., Price, K.J., et al. (2015) Place of Death among Hospitalized Patients with Cancer at the End of Life. Journal of Palliative Medicine, 18, 667-676. https://doi.org/10.1089/jpm.2014.0389

[14] Dy, S.M., Wolff, J.L. and Frick, K.D. (2007) Patient Characteristics and End-of-Life Health Care Utilization among Medicare Beneficiaries in 1989 and 1999. Medical Care, 45, 926-930. https://doi.org/10.1097/MLR.0b013e31812714a5

[15] Mohsen, H., Haddad, P., Allam, A. and Hassan, A. (2014) Patterns in Place of Cancer Death in the State of Qatar: A Population-Based Study. PLoS ONE, 9, e109615. https://doi.org/10.1371/journal.pone.0109615

[16] Newman, A., Thompson, J. and Chandler, E.M. (2013) Continuous Care: A Home Hospice Benefit. Clinical Journal of Oncology Nursing, 17, 19. https://doi.org/10.1188/13.CJON.19-20

[17] Beernaert, K., Deliens, L., Pardon, K., Van den Block, L., et al. (2015) What Are Physicians' Reasons for Not Referring People with Life-Limiting Illnesses to Specialist Palliative Care Services? A Nationwide Survey. PLoS ONE, 10, e0137251. https://doi.org/10.1371/journal.pone.0137251

[18] Cohen, J., Pivodic, L., Miccinesi, G., Onwuteaka-Philipsen, B.D., et al. (2015) International Study of the Place of Death of People with Cancer: A Population-Level Comparison of 14 Countries across 4 Continents Using Death Certificate Data. British Journal of Cancer, 113, 1397-1404. 
[19] National Cancer Center (2015) Cancer Facts and Figures 2014 in the Republic of Korea. Seoul.

[20] Sekiguchi, K., Bell, C.L., Masaki, K.H. and Fischberg, D.J. (2014) Factors Associated with In-Hospital Death by Site of Consultation among Elderly Inpatients Receiving Pain and Palliative Care Consultations. Journal of Palliative Medicine, 17, 1353-1358. https://doi.org/10.1089/jpm.2013.0596

[21] Teno, J., Lynn, J., Connors, A.F., Wenger, N., Phillips, R.S., Alzola, C., Murphy, D.P., Desbiens, N. and Knaus, W.A. (1997) The Illusion of End-of-Life Resource Savings with Advance Directives. Support Investigators. Study to Understand Prognoses and Preferences for Outcomes and Risks of Treatment. Journal of the American Geriatrics Society, 45, 513-518.

[22] Tangeman, J.C., Rudra, C.B., Kerr, C.W. and Grant, P.C. (2014) A Hospice-Hospital Partnership-Reducing Hospitalization Costs and 30-Day Readmissions among Seriously Ill Adults. Journal of Palliative Medicine, 17, 1005-1010. https://doi.org/10.1089/jpm.2013.0612

[23] Morrison, H. (2005) Health Care System Factors Affecting End-of-Life Care. Journal of Palliative Medicine, 8, S79-S87. https://doi.org/10.1089/jpm.2005.8.s-79

[24] Hebert, R.S., Dang, Q. and Schulz, R. (2007) Religious Beliefs and Practices Are Associated with Better Mental Health in Family Caregivers of Patients with Dementia: Findings from the REACH Study. The American Journal of Geriatric Psychiatry, 15, 292-300. https://doi.org/10.1097/01.JGP.0000247160.11769.ab

[25] Tilden, V.P., Tolle, S.W., Drach, L.L. and Perrin, N.A. (2004) Out-of-Hospital Death: Advance Care Planning, Decedent Symptoms, and Caregiver Burden. Journal of the American Geriatrics Society, 52, 532-539. https://doi.org/10.1111/j.1532-5415.2004.52158.x

[26] Teno, J.M., Weitzen, S., Fennell, M.L. and Mor, V. (2001) Dying Trajectory in the Last Year of Life: Does Cancer Trajectory Fit Other Diseases? Journal of Palliative Medicine, 4, 457-464. https://doi.org/10.1089/109662101753381593

[27] Teno, J.M., Bynum, J.P., Leland, N.E., Miller, S.C., Morden, N.E., Scupp, T., Goodman, D.C. and Mor, V. (2013) Change in End-of-Life Care for Medicare Beneficiaries: Site of Death, Place of Care, and Health Care Transitions in 2000, 2005, and 2009. JAMA, 309, 470-477. https://doi.org/10.1001/jama.2012.207624

[28] Kim, J.H., Kim, S.M., Joo, J.S. and Lee, K.S. (2012) Factors Associated with Medical Cost among Patients with Terminal Cancer in Hospice Units. Journal of Palliative Care, 28, 5-12. https://doi.org/10.1177/082585971202800102

[29] Sasao, S., Tanabe, K., Morita, T., Takahashi, T., et al. (2015) Facility-Related Factors Influencing the Place of Death and Home Care Rates for End-Stage Cancer Patients. Journal of Palliative Medicine, 18, 691-696. https://doi.org/10.1089/jpm.2014.0384

[30] Fukui, S., Fujita, J., Tsujimura, M., Sumikawa, Y. and Hayashi, Y. (2011) Predictors of Home Death of Home Palliative Cancer Care Patients: A Cross-Sectional Nationwide Survey. International Journal of Nursing Studies. International Journal of Nursing Studies, 48, 1393-1400. https://doi.org/10.1016/j.ijnurstu.2011.05.001

[31] Nakamura, S., Kuzuya, M., Funaki, Y., Matsui, W. and Ishiguro, N. (2010) Factors Influencing Death at Home in Terminally Ill Cancer Patients. Geriatrics \& Gerontology International, 10, 154-160.

[32] Lee, L.C., Hu, C.C., Loh, el-W. and Hwang, S.F. (2014) Factors Affecting the Place of Death among Hospice Home Care Cancer Patients in Taiwan. American Journal of Hospice and Palliative Medicine, 31, 300-306. https://doi.org/10.1177/1049909113487427 
[33] Alonso-Babarro, A., Bruera, E., Varela-Cerdeira, M., Boya-Cristia, M.J., Madero, R., Torres-Vigil, I., De Castro, J. and González-Barón, M. (2011) Can This Patient Be Discharged Home? Factors Associated with At-Home Death among Patients with Cancer. Journal of Clinical Oncology, 29, 1159-1167. https://doi.org/10.1200/JCO.2010.31.6752

[34] Yun, Y.H., Lim, M.K., Choi, K.S. and Rhee, Y.S. (2006) Predictors Associated with the Place of Death in a Country with Increasing Hospital Deaths. Palliative Medicine, 20, 455-461. https://doi.org/10.1191/0269216306pm1129oa

[35] Lee, Y.S. (2013) The Past, Present and Future Strategies of Korean Social Work in Hospice and Palliative Care. Korean Journal of Hospice and Palliative Care, 16, 65-73. https://doi.org/10.14475/kjhpc.2013.16.2.065

[36] Park, C.S., Yoon, S.J. and Jung, Y. (2013) The Status of Home-Based Hospice Care in Korea. Korean Journal of Hospice and Palliative Care, 16, 98-107.

[37] Rhee, Y. (2015) Hospice and Palliative Care Services in South Korea Supported by the National Health Insurance (NHI) Program. Health, 7, 689-695. https://doi.org/10.4236/health.2015.76082

[38] Health Insurance and Research Assessment (HIRA) (2016) The Guidelines for Pilot Project: Pricing and Services items in Home-Based Hospice and Palliative Care Services. Health Insurance and Research Assessment, Seoul.

\section{List of Abbreviations}

NHI: National Health Insurance

NHIS: The National Health Insurance Service

CT: Computed Tomography

PET: Polyethylene Terephthalate

MRI: Magnetic Resonance Imaging

ER: Emergency Room

CAD: Coronary Artery Disease

COPD: Chronic Obstructive Pulmonary Disease

CHF: Congestive Heart Failure

CPR: Cardiopulmonary Resuscitation

OR: Odds Ratio 